\title{
Weekly cisplatin, epirubicin, and paclitaxel with granulocyte colony-stimulating factor support vs triweekly epirubicin and paclitaxel in locally advanced breast cancer: final analysis of a sicog phase III study
}

\section{G Frasci",', G D'Aiuto', P Comella', R Thomas', G Botti', M Di Bonito', V De Rosa', G lodice', MR Rubulotta' and G Comella' for the Southern Italy Cooperative Oncology Group (SICOG)- Italy}

'Giuseppe Frasci, Division of Medical Oncology A, National Tumor Institute, via Mariano Semmola 80 I 3 I, Naples, Italy

The present study aimed at evaluating whether a weekly cisplatin, epirubicin, and paclitaxel (PET) regimen could increase the pathological complete response ( $\mathrm{PCR}$ ) rate in comparison with a tri-weekly epirubicin and paclitaxel administration in locally advanced breast cancer ( $L A B C$ ) patients. Patients with stage IIIB disease were randomised to receive either 12 weekly cycles of cisplatin $30 \mathrm{mg} \mathrm{m}^{-2}$, epirubicin $50 \mathrm{mg} \mathrm{m}^{-2}$, and paclitaxel $120 \mathrm{mg} \mathrm{m}^{-2}$ (PET) plus granulocyte-colony stimulating factor support, or four cycles of epirubicin $90 \mathrm{mg} \mathrm{m}^{-2}+$ paclitaxel $175 \mathrm{mg} \mathrm{m}^{-2}(\mathrm{ET})$ every 3 weeks. Overall, 200 patients $(\mathrm{PET} / \mathrm{ET}=100 / 100)$ were included in this study. A pCR in both breast and axilla occurred in 16 (16\%) PET patients and in six (6\%) ET patients $(P=0.02)$. The higher activity of PET was evident only in ER negative (27.5 vs 5.4\%; $P=0.026)$, and in HER/neu positive $(3 \mathrm{I}$ vs $5 \%$; $P=0.037)$ tumours. The two arms yielded similar PCR rate in ER positive (PET/ET $=7.5 / 7.1 \%)$ and $\mathrm{HER} /$ neu negative $(\mathrm{PET} / \mathrm{ET}=10 / 6 \%)$ patients. At a 39 months median follow-up, 70 patients showed a progression or relapses (PET, 32 vs ET, 38). Anaemia, mucositis, peripheral neuropathy, and gastrointestinal toxicity were substantially more frequent in the PET arm. The PET weekly regimen is superior to ET in terms of $\mathrm{PCR}$ rate in LABC patients with ER negative and/or HER2 positive tumours Mature data in terms of disease-free and overall survival are needed to ascertain whether this approach could improve the prognosis of these subsets of $\angle A B C$ patients.

British Journal of Cancer (2006) 95, I005-1012. doi: I0.1038/sj.bjc.6603395 www.bjcancer.com

(c) 2006 Cancer Research UK

Keywords: paclitaxel; epirubicin; cisplatin; weekly administration; LABC; randomised trial

The role of chemotherapy in improving the prognosis of locally advanced breast cancer (LABC) patients is widely recognised. High objective response rates can be achieved with standard combination chemotherapy, rendering in most cases the tumour operable at the end of the treatment (Hortobagyi and Buzdar, 1997). Primary chemotherapy is also becoming very popular in patients with operable disease at diagnosis, since it permits a substantial increase in the rate of breast-sparing surgery, and allows an early pathological evaluation of the effect of treatment. The absence of residual tumour in both breast and axilla is associated with a much lower risk of relapse (Chollet et al, 2002; Faneyete et al, 2003). Unfortunately, the chance of achieving such a goal in women with LABC is low. Indeed, in the NSABP-B18 trial, the pCR rate after primary chemotherapy was $<3 \%$ in women with T3 (operable) disease (Fisher et al, 1997). During the last decade, several new drugs with different mechanisms of action have been introduced into clinical practice. Among them, the taxanes (paclitaxel and docetaxel) have raised

*Correspondence: Dr G Frasci; E-mail: giuseppe.frasci@libero.it Received 3 April 2006; revised 4 September 2006; accepted 4 September 2006 great enthusiasm, and have been rapidly incorporated in combination regimens (Dombernowsky et al, 1996; Moliterni et al, 1997).

Phase III trials comparing taxane- $v s$ non taxane-including regimens, have been carried out in breast cancer patients with loco-regional disease. Both in the NSABP-B27 (Bear et al, 2003) and in the Aberdeen (Smith et al, 2002) studies, the addition of docetaxel resulted in a two-times greater pCR rate in comparison with the standard arm. Notably, a substantial proportion of patients in the Aberdeen trial had LABC. French investigators (Dieras et al, 2004) reported a significantly higher pCR rate with primary doxorubicin plus paclitaxel in comparison with a standard doxorubicin plus cyclophosphamide regimen.

A better therapeutic index has been recently hypothesised for the weekly paclitaxel administration. In an MDACC trial (Green et al, 2005), 258 patients with operable disease were randomised to receive either weekly or every 3 weeks paclitaxel followed by four FAC (5-fluorouracil, doxorubicin and cyclophosphamide cycles before surgery. The pCR rate in the weekly arm was twice that of the tri-weekly arm (28.8 vs $13.6 \%)$.

In the last few years, increasing interest has arisen about the role of platinum compounds in the treatment of breast cancer patients. Several cisplatin-based regimens have been tested in the 
neo-adjuvant setting, showing high anti tumour activity (Orlando et al, 2001; Ezzat et al, 2004). In the mid-nineties, we started the assessment of a weekly triplet regimen including cisplatin, epirubicin, and paclitaxel (PET regimen) in breast cancer patients (Frasci et al, 1999). This weekly approach was initially tested in a phase II study in women with LABC (Frasci et al, 2000). The promising activity data we observed (overall response rate, $90 \%$, and $\mathrm{pCR}$ rate, $12 \%$ ) prompted us to start the present phase III trial, which aimed at comparing this regimen with a tri-weekly epirubicin plus paclitaxel administration.

\section{METHODS}

\section{Eligibility criteria}

Patients with histological proven locally advanced (T4 a-d and/or N2 fixed to other structures) breast cancer with no prior chemotherapy were eligible. Other selection criteria were: age $<75$, ECOG performance status $\leqslant 2$, adequate bone marrow (ANC $\geqslant 2.0 \times 10^{9} \mathrm{l}^{-1}$, platelet count $\geqslant 100 \times 10^{9} \mathrm{l}^{-1}$, and haemoglobin level $\geqslant 100 \mathrm{gl}^{-1}$ ), liver (bilirubin level $<1.5$ the upper normal limit [UNL], AST and/or ALT $<3 \times$ UNL, prothrombin time $<1.5$ times control), renal (creatinine clearance $\geqslant 60 \mathrm{ml} \mathrm{min}^{-1}$ ) and cardiac functions (left ventricular ejection fraction $>50 \%$, absence of severe cardiac arrhythmia or heart failure, second- or third-degree heart block or acute myocardial infarction within 4 months prior to study entry). Previous or concurrent malignancy, were also considered as exclusion criteria, except for inactive nonmelanoma skin cancer, and in situ carcinoma of the cervix. All patients gave their written informed consent, and the trial was approved by the Independent Ethical Committee of the National Tumour Institute of Naples.

\section{Pretreatment evaluation}

Within 4 weeks before starting chemotherapy, all patients underwent the following studies: complete history and physical examination, ECG and bi-dimensional echocardiography, mammography, chest X-ray, liver ultrasonography, radionuclide bone scan (with X-ray evaluation of suspicious bone segments), and CT or MRI of the brain in the case of suspected brain involvement. Laboratory investigation included a complete blood cell count with white blood cell (WBC) differential and platelet count, BUN, creatinine, bilirubin, SGOT, alkaline phosphatase, lactate dehydrogenase, prothrombin time, partial thromboplastin and thrombin time, urinalysis. A core biopsy of the primary tumour was also performed with the immunohistochemical assessment of the main prognostic variables (steroid hormones receptors, Ki67, HER2/ neu). The HER2/neu was measured by using the monoclonal antibodies Mab 1 and CB11 on $3 \mu \mathrm{m}$ sections of paraffin-embedded tumour samples.

\section{Treatment}

All eligible patients were randomly allocated to receive: (arm A) epirubicin $50 \mathrm{mg} \mathrm{m}^{-2}$ as an i.v. bolus, followed by paclitaxel $120 \mathrm{mg} \mathrm{m}^{-2}$ as a $1-\mathrm{h}$ infusion, and cisplatin $30 \mathrm{mg} \mathrm{m}^{-2}$ as a $30 \mathrm{~min}$ infusion, weekly for a maximum of 12 cycles. Recombinant human G-CSF $300 \mu \mathrm{g} \mathrm{day}^{-1}$ was also given subcutaneously on days 3-5 of each week. Short-term forced hyperhydration (11 of saline over $2 \mathrm{~h}$ ), and prophylaxis for nausea/vomiting (HT3 receptor antagonists) were also performed. Prophylaxis for hypersensitivity reactions consisted of dexamethasone $8 \mathrm{mg}$ i.v. and promethazine $50 \mathrm{mg}$ i.m. plus ranitidine $50 \mathrm{mg}$ i.v. $30 \mathrm{~min}$ before paclitaxel administration; or ( $\mathrm{arm} \mathrm{B}$ ) epirubicin $90 \mathrm{mg} \mathrm{m}^{-2}$ as an i.v. bolus, followed by paclitaxel $175 \mathrm{mg} \mathrm{m}^{-2}$ as a 1-h infusion, every 3 weeks for a maximum of four cycles. The same antiemetic and antihypersensitivity procedures adopted for arm A were performed.
Within 4 weeks from the end of chemotherapy patients underwent surgery. Breast sparing surgery was performed whenever feasible. It consisted of quadrantectomy together with standard level I and II axillary lymph-node dissection.

Four cycles of CMF were delivered after surgery, in patients whose pathologic assessment showed less than four involved axillary lymph nodes; whereas four FEC (epirubicin $60 \mathrm{mg} \mathrm{m}^{-2}$ instead of methotrexate) cycles were administered in those women showing four or more involved axillary nodes. On completion of postoperative chemotherapy, radiotherapy was performed on all patients who underwent conservative surgery, and to those who underwent mastectomy and had more than three axillary nodes involved, muscle, skin and/or nipple involvement, or had G3 tumour at diagnosis.

Hormone treatment was also given on completion of postoperative chemotherapy. LH-RH analogue for 2 years together with tamoxifen for 5 years was administered in premenopausal, and 5-year tamoxifen was given in postmenopausal women.

\section{Dose adjustments according to toxicity}

Arm A: chemotherapy was given at full doses if neutrophil count was $\geqslant 1.5 \times 10^{9} 1^{-1}$, and platelet count was $\geqslant 100 \times 10^{9} 1^{-1}$. Doses were reduced by $50 \%$ if neutrophil count was $<1.5 / 10^{9}$ and $\geqslant 1.0 /$ $10^{9} 1^{-1}$, or platelet count was $<100 \times 10^{9} 1^{-1}$ and $\geqslant 75 / 10^{9} 1^{-1}$. In the case of grade $\geqslant 3$ neutropenia, grade $\geqslant 2$ thrombocytopenia, or grade $>1$ nonhaematological toxicity the treatment was always omitted. In the presence of grade 4 neutropenia, febrile neutropenia, grade 4 thrombocytopenia, grade 4 anaemia, grade 3-4 nonhaematological toxicity (except for alopecia) doses were reduced by $25 \%$ in the subsequent administrations. G-CSF was allowed in the presence of grade 4 neutropenia of any duration, or neutropenic fever, or if grade 3 neutropenia persisted for 2 weeks after the scheduled time of recycling. The treatment was definitively discontinued if chemotherapy could not be delivered 3 weeks after the scheduled time of recycling.

Arm B: chemotherapy was given at full doses for neutrophil count $\geqslant 2000 \mathrm{~mm}^{-3}$, and platelet count $\geqslant 100000 \mathrm{~mm}^{-3}$. If neutrophil count was $\geqslant 1500 \mathrm{~mm}^{-3}$ persisted after a 1-week delay, chemotherapy was delivered at $75 \%$ of the planned dose.

In the presence of grade 4 neutropenia, febrile neutropenia, grade 4 thrombocytopenia, grade 4 anaemia, grade 3-4 nonhaematological toxicity (except for alopecia) doses were reduced to $75 \%$ in the subsequent administrations. G-CSF was allowed in the presence of grade 4 neutropenia of any duration, or neutropenic fever, or if grade 3 neutropenia persisted for 2 weeks after the scheduled time of recycling. The treatment was definitively discontinued if chemotherapy could not be delivered three weeks after the scheduled time of recycling.

\section{Toxicity and response evaluation criteria}

Toxicity was assessed at the weekly visits and recorded using the NCI-CTC version 3.0. Complete blood cell and WBC count were performed once a week in all patients, and every other day in cases of grade 4 haematological toxicity. Bidimensional echocardiography was performed on completion of treatment.

Clinical tumour response was assessed within 2 weeks from the end of chemotherapy, and classified according to standard WHO criteria (Miller et al, 1981). Clinical examination, mammography, breast ultrasonography were performed to assess the regression of the tumour in both breast and axilla. In addition, chest X-ray, and abdomen ultrasonography were also performed, in order to exclude the presence of distant metastases.

For pathologic assessment of response, the amount of residual epithelial neoplastic cells in the tumour mass, and the location of malignant component (invasive $v s$ intraductal) were taken into 
account (Chevallier et al, 1993). Response in the breast was scored as follows: class I (absence of residual malignant epithelial cells), class II (persistence of only in situ residual malignant component), class III (only focal invasive tumour residuals), class IV (no substantial modifications in the tumour mass). Patients showing a class I or II response in the breast, together with absence of axillary involvement were considered as PCR.

\section{End points and statistical considerations}

The pCR rate was the main end point. We set as 5\% the pCR rate expected with the administration of epirubicin plus paclitaxel, and we hypothesised a $10 \%$ increase with the PET regimen. To have an $80 \%$ power to demonstrate such a difference with an alpha error $<5 \%$, at least 90 patients per arm were required (Casagrande et al, 1978).

Progression-free survival (PFS) and overall survival (OS) were also analysed. PFS was considered as the interval between randomisation and documentation of progressive disease or relapse (in surgically treated patients). Overall survival was considered as the interval between randomisation and death by any cause. All time-dependent curves were estimated by the Kaplan Meier method (Kaplan and Meier, 1958). All patients were included in the analysis of response and survival on an intent to treat basis'.

\section{RESULTS}

\section{Demographics}

Between May 1999 and November 2004, 200 consecutive women with $\mathrm{LABC}$ were enrolled into this study. Table 1 outlines the main patient characteristics. Overall, 136 patients had T4a-b-c disease, while 47 women had inflammatory carcinoma, and 17 N2 disease. Patients (144) had ductal carcinoma, and oestrogen or progesterone receptors were positive in 110 and 103 patients, respectively. Patients (43) were HER2 + .

\section{Compliance to proposed treatment}

Women (13) in the PET and seven women in ET arm did not complete the planned number of chemotherapy cycles. Early disease progression was the cause of treatment discontinuation in three PET and four ET patients. The remaining early discontinuations were a consequence of severe toxicity or patient's refusal: in the PET arm, reasons for withdrawal were: severe emesis in two cases, persistence of severe mucositis in one case, severe peripheral neuropathy in two cases, severe fatigue in three cases, and patient's refusal in two cases. In the ET arm, severe emesis (one case), and fatigue (two cases) forced the treatment interruption.

A treatment delay due to persistence of haematological toxicity or grade $>1$ non haematological toxicity on day of recycling occurred in 18 and 11 patients in PET and ET arm, respectively. A dose reduction was performed at least once in 35 PET and 21 ET patients, respectively. In all, 71 PET and 83 ET patients actually received $\geqslant 80 \%$ of the planned dose-intensity (Figure 1 ).

\section{Response}

All the 200 enrolled patients were included into the response analysis on an 'intent to treat basis'. Table 2 summarises clinical response data. In the PET arm, 28 complete and 60 partial responses were recorded at clinical restaging. An additional nine patients showed a minor regression or stabilisation of the tumour. In the ET arm, a clinically complete or partial response occurred in 19 and 59 patients, respectively. An additional 18 women showed a minor response or no change.
Table I Demographics

\begin{tabular}{|c|c|c|c|}
\hline Characteristic & $\begin{array}{l}\text { PET (100 } \\
\text { patients) }\end{array}$ & $\begin{array}{c}\text { ET ( } 100 \\
\text { patients) }\end{array}$ & $\begin{array}{c}\text { Total (200 } \\
\text { patients) }\end{array}$ \\
\hline \multicolumn{4}{|l|}{ Age } \\
\hline Median (range) & $53(27-73)$ & $54(30-72)$ & $54(27-73)$ \\
\hline$<65 / \geqslant 65$ & $52 / 48$ & $49 / 5 i$ & $101 / 99$ \\
\hline \multicolumn{4}{|l|}{ Stage } \\
\hline T4 No & 12 & 14 & 26 \\
\hline T4 NI & 79 & 78 & 157 \\
\hline Any $T \mathrm{~N} 2$ & 9 & 8 & 17 \\
\hline \multicolumn{4}{|l|}{ Breast tumour } \\
\hline $\mathrm{T} 4 \mathrm{a}-\mathrm{c} / \mathrm{d}$ & $67 / 24$ & $69 / 23$ & | 36/47 \\
\hline \multicolumn{4}{|l|}{ Histology } \\
\hline Ductal & 71 & 73 & 144 \\
\hline Lobular & 19 & 18 & 37 \\
\hline Mixed & 4 & 4 & 8 \\
\hline Mucinous & 3 & 2 & 5 \\
\hline Other & 3 & 3 & 6 \\
\hline \multicolumn{4}{|l|}{ Grading } \\
\hline I & 9 & 12 & 21 \\
\hline$\|$ & 33 & 30 & 63 \\
\hline III & 55 & 54 & 109 \\
\hline Unknown & 3 & 4 & 7 \\
\hline \multicolumn{4}{|l|}{ Menopausal status } \\
\hline Pre-/postmenopausal & $33 / 67$ & $31 / 69$ & $64 / 136$ \\
\hline \multicolumn{4}{|l|}{ Hormone receptors status } \\
\hline ER: yes/no/unknown & $53 / 40 / 7$ & $57 / 37 / 6$ & $110 / 77 / 13$ \\
\hline PgR: yes/no/unknown & $50 / 43 / 7$ & $53 / 41 / 6$ & $103 / 84 / 13$ \\
\hline \multicolumn{4}{|l|}{ HER/neu status } \\
\hline Pos/neg/unknown & 23/68/9 & $20 / 68 / 12$ & $43 /|36 / 2|$ \\
\hline
\end{tabular}

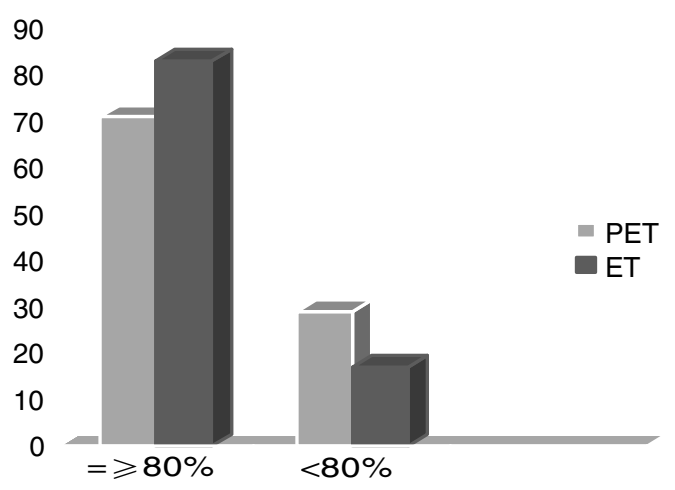

Figure I Relative dose Intensity.

According to receptor status, a clinical complete response occurred in $19 / 40(47.5 \%)$ ER negative and $8 / 53$ (15\%) ER positive patients (one $\mathrm{CR}$ in a woman with hormone receptor status unknown) in the PET arm. In the ET arm, a clinical complete response occurred in 6/37 (16.2\%) ER negative, and 12/57 (21\%) ER positive patients (one CR in a patient with unknown hormone receptor status). Regarding HER2/neu status, 9/23 (40\%) HER2 + and 16/68 (23.6\%) HER2 - achieved a complete response in the PET arm, as compared to $7 / 20$ (35\%) HER2 + and 10/68 (14.7\%) HER 2 - in ET arm (Table 3).

Overall, 186 women (PET 94, ET 92) underwent surgery. Breast sparing surgery was performed in a total of 37 patients (PET 23, ET 14). Table 4 summarises the pathological response data. In all, 
22 patients (PET 13, ET 9) showed absence of residual malignant epithelial cells, either invasive or intraductal, in the breast specimen. Only in situ residual tumour cells were found in additional 14 women (PET 9, ET 5). Therefore, the pCR rate in the breast was $22 \%$ in PET and $14 \%$ and ET arm, respectively. A pPR in the breast (i.e., only focal invasive tumour residuals in the removed breast tissue) was recorded in 38 PET and 26 ET patients, respectively. Overall, 59 women (PET 35, ET 24) showed negative axilla. Among the 141 patients with persistence of tumour in the axilla, 60 (PET 33, ET 27) had one to three lymph nodes involved, and 81 (PET 32, ET 49) four or more nodes.

In all, 22 patients (PET 16, ET 6) showed an absence of invasive tumour in both breast and axilla; the pCR rate being 16 and $6 \%$ in PET and ET arm, respectively $(P=0.02)$. In the PET arm, 11 pCRs were observed among 40 ER negative tumours $(27.5 \%)$ as compared to four pCRs among 53 ER positive (7.5\%), and one among the seven with unknown hormone receptor status (14.3\%). In the ET arm, two pCRs were registered in the 37 ER negative (5.4\%) and four pCRs in the 57 ER positive tumours (7.1\%). One out of four women with ER positive tumour, achieving a pCR, had negative PgR. All 11 ER negative tumours who achieved a pCR in the PET arm, were PgR negative too. If a comparison between the two arms is made taking into account the hormone receptor status, the advantage of PET treatment is highly evident in ER negative patients (PET 27.5 vs ET 5.4\%; $P=0.026$ ), while it did not appear in the ER positive subgroup (PET 7.5 vs ET 7\%) (Table 5).

Table 2 Clinical response according to treatment ARM

\begin{tabular}{lclcc}
\hline & PET no. (\%) & & ET no. (\%) & Total no. (\%) \\
\hline Complete & $28(28)$ & P N NS & $19(19)$ & $47(23.5)$ \\
Partial & $60(60)$ & & $59(59)$ & $119(59.5)$ \\
MR/NC & $9(9)$ & & $18(18)$ & $27(13.5)$ \\
PD & $3(3)$ & & $4(4)$ & $7(3.5)$ \\
\hline
\end{tabular}

Table 3 Complete response according to hormone receptors and her/ neu status

\begin{tabular}{|c|c|c|c|c|}
\hline & PET no. (\%) & & ET no. (\%) & Total no. (\%) \\
\hline \multicolumn{5}{|c|}{ Hormone receptors } \\
\hline ER- & $19 / 40(47.5)$ & $P<0.05$ & $6 / 37(16.2)$ & 25/77 (32.5) \\
\hline $\begin{array}{l}\text { ER+ } \\
\text { Unknown }\end{array}$ & $\begin{array}{l}8 / 53(15) \\
1 / 7(14.5)\end{array}$ & $P=N S$ & $\begin{array}{l}12 / 57(2 \mid) \\
1 / 6(16.5)\end{array}$ & $\begin{array}{l}20 / 110(18) \\
2 / 13(15.4)\end{array}$ \\
\hline \multicolumn{5}{|l|}{ HER/neu } \\
\hline+ & $9 / 23(40)$ & $P=N S$ & $7 / 20$ (35) & $16 / 43(37.2)$ \\
\hline- & 16/68 (23.6) & $P=N S$ & $10 / 68(14.7)$ & $26 / 136(19)$ \\
\hline Unknown & $3 / 9(33.3)$ & & $2 / 12(16.7)$ & $5 / 21(24)$ \\
\hline
\end{tabular}

Table 4 Pathological assessment according to treatment arm

\begin{tabular}{|c|c|c|c|c|}
\hline & PET no. (\%) & & ET no. (\%) & Total no. (\%) \\
\hline \multicolumn{5}{|l|}{ Breast } \\
\hline Class 1 & $13(13)$ & $P=N S$ & $9(9)$ & $22(11)$ \\
\hline Class II & $9(9)$ & $P=N S$ & $5(5)$ & $14(7)$ \\
\hline Class III & $38(38)$ & & $26(26)$ & $64(32)$ \\
\hline Class IV & $40(40)$ & & $60(60)$ & $100(50)$ \\
\hline \multicolumn{5}{|l|}{ Axilla } \\
\hline NO & $35(35)$ & & $24(24)$ & $59(29.5)$ \\
\hline $\mathrm{NI}-3$ & $33(33)$ & & $27(27)$ & $60(30)$ \\
\hline$N>3$ & $32(32)$ & & $49(49)$ & $81(40.5)$ \\
\hline pCR (class I+\| and N0) & $16(16)$ & $P=0.02$ & $6(6)$ & $22(11)$ \\
\hline
\end{tabular}

Eight pCRs were observed in the 43 patients (18.6\%) HER2/neu positive, as compared to $11 / 136(8 \%)$ HER2 negative cases $(3 / 21$ patients with HER2/neu unknown had a pCR). Also in this case the superiority of the PET regimen was evident only in those patients with HER2 positive tumour (31 vs 5\%; $P=0.037$ ) (Table 5).

After a median follow-up of 39 (range, 4-70) months, 70 patients had progressed or relapsed (PET 32, ET 38), and the median PFS was 38.5 and 40.1 months in PET and ET arm, respectively. Five-year projected PFS probability was 30 and $25 \%$ for PET and ET, respectively (Figure 2).

Seven (PET 4, ET 3) of the 22 patients showing a pCR had relapsed at the time of the present analysis (Figure 3 ).

Overall, 35 out of 77 patients with ER negative tumour (PET 16, ET 19) had progression or relapse at the time of the present analysis, as compared to 31 (PET 14, ET 17) out of 110 patients with ER positive tumour; median PFS being 30.1 and 47.6 months, respectively (Figure 4). Median PFS times in ER positive patients were 38.2 months and 40.7 months for PET and ET, respectively; while they were 32 and 34 months, respectively, in ER negative patients (Figures 5-6). Four failures occurred in the 13 patients with unknown receptor status.

Overall, 19 (PET 8, ET 11) of the 43 patients with HER2 positive tumour had progressed or relapsed, median PFS being 25.5 months. A total of 43 (PET 20, ET 23) failures had occurred in the 136 HER2 negative patients, median PFS being 44.7 months (Figure 7).

Overall, 26 deaths (PET 10, ET 16) had occurred at the time of the present analysis. Median survival had not been reached in both arms.

\section{Toxicity}

A total of 1122 PET courses and 386 ET courses were delivered and analysed for toxicity. No toxic deaths were observed in either arms.

Table 5 Pathological complete response according to pretreatment features

\begin{tabular}{lccc}
\hline & PET no. pCR (\%) & ET no. pCR (\%) & Total no. pCR (\%) \\
\hline ER- & $1 / / 40(27.7)^{*}$ & $2 / 37(5.4)$ & $13 / 77(17)$ \\
ER+ & $4 / 53(7.5)$ & $4 / 57(7.1)$ & $8 / 110(7)$ \\
Unknown & $\mid / 7(\mid 4)$ & $0 / 6$ & $1 / 13(8)$ \\
HER pos & $7 / 23(3 \mid)^{\#}$ & $1 / 20(5)$ & $8 / 43(18.6)$ \\
HER neg & $7 / 68(\mid 0)$ & $4 / 68(6)$ & $\mid 1 / 136(8)$ \\
Unknown & $2 / 9(22)$ & $1 / 12(8)$ & $3 / 21(14)$ \\
\hline
\end{tabular}

*PET vs ET: $P=0.026 .{ }^{\#} \mathrm{PET}$ vs ET; $P=0.037$.

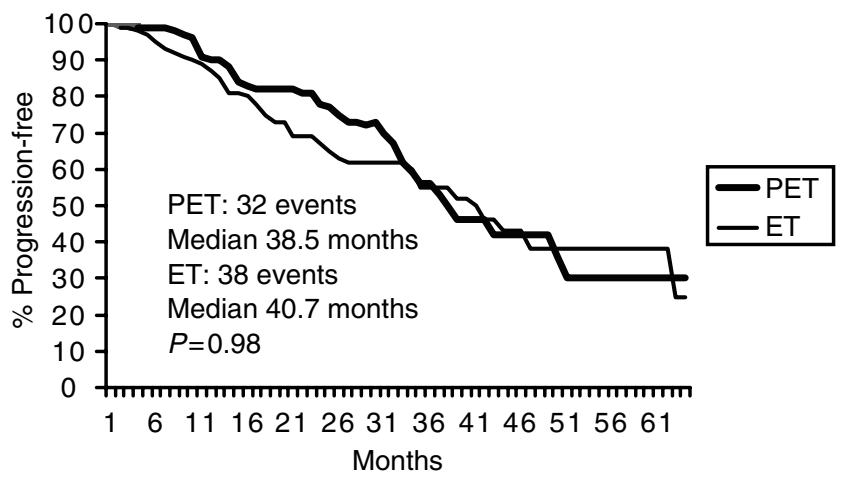

Figure 2 Progression free survival. 


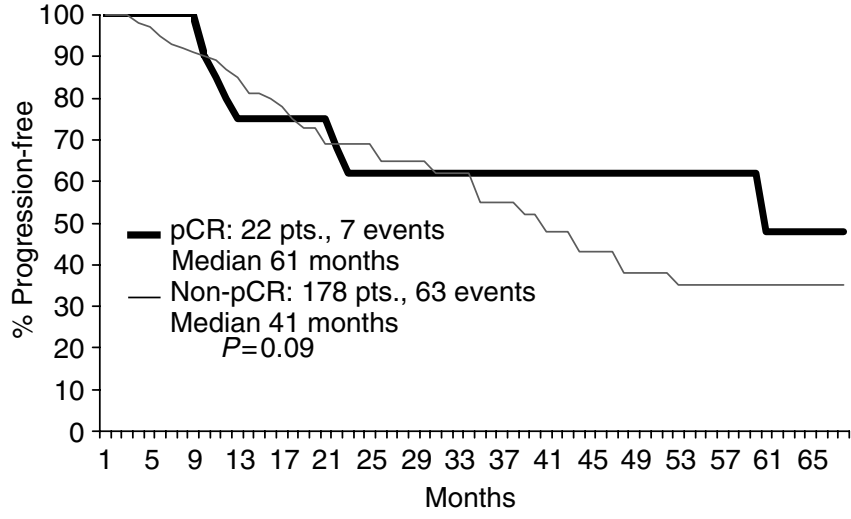

Figure 3 DFS according to pathological response.

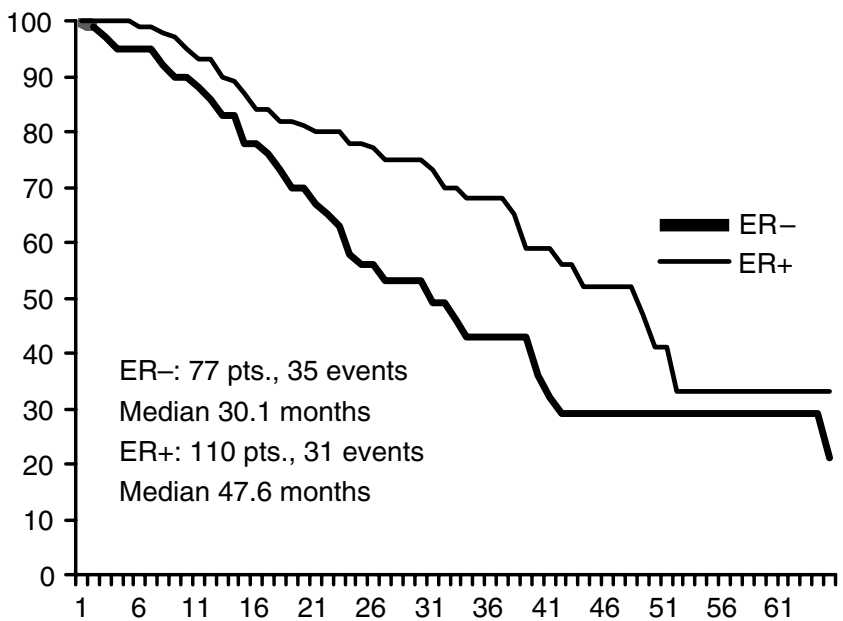

Figure 4 PFS according to ER status.

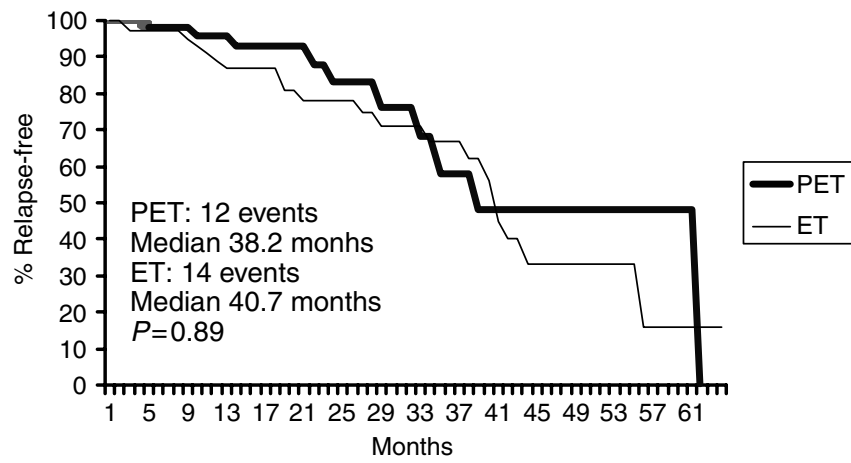

Figure 5 PFS in ER/PgR positive.

Table 6 shows the number of patients in each arm who experienced at least one grade $\geqslant 3$ toxicity.

Haematological toxicity was not substantially different in the two arms, except for anaemia. Overall 45 patients in PET and 38 in ET arm experienced a grade $\geqslant 3$ neutropenia. Grade 4 occurred in 17 and 18 cases, respectively. Neutropenic fever was observed in three PET and four ET patients. Severe thrombocytopenia was almost anecdotic, grade 3 or 4 occurring in only six patients (four PET and two ET). Only one case of grade 4 thrombocytopenia was

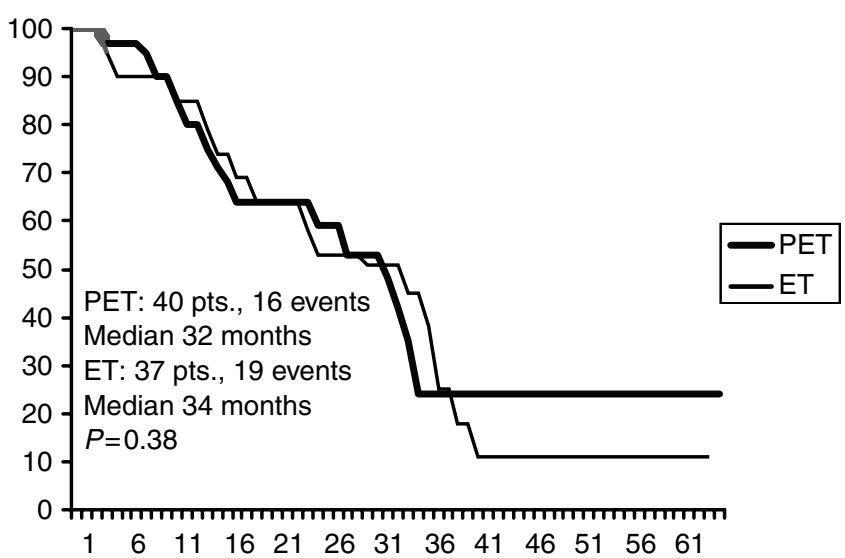

Figure 6 PFS in ER negative patients.

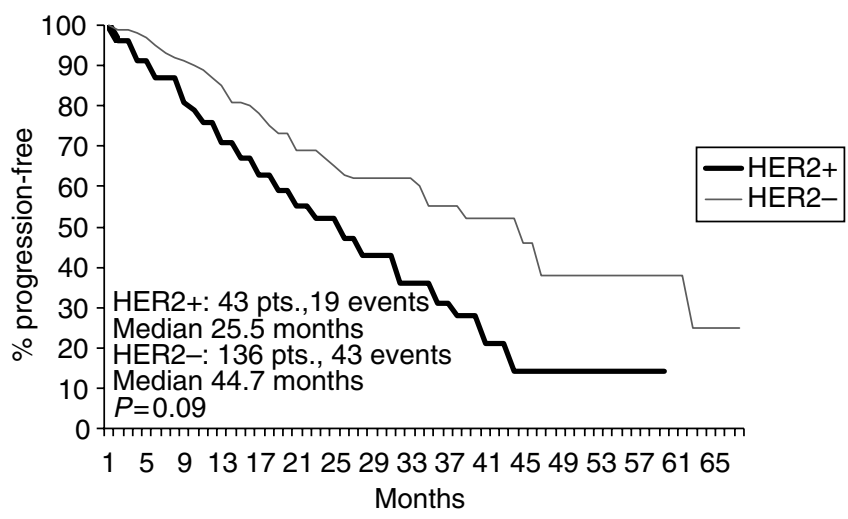

Figure 7 PFS according to HER2 status.

Table 6 Who grade 3-4 toxicity in the two arms

\begin{tabular}{lcc}
\hline & $\begin{array}{c}\text { PET (1 00) } \\
\text { No. of patients (\%) }\end{array}$ & $\begin{array}{c}\text { ET (1 00) } \\
\text { No. of patients (\%) }\end{array}$ \\
\hline Neutropenia & $45(45)$ & $38(38)$ \\
Sepsis & $3(3)$ & $4(4)$ \\
Thrombocytopenia & $4(4)$ & $2(2)$ \\
Anaemia & $13(13)$ & $1(1)$ \\
Nausea & $9(9)$ & $6(6)$ \\
Vomiting & $6(6)$ & $4(4)$ \\
Loss of appetite & $13(10)$ & $5(5)$ \\
Diarrhoea & $15(15)$ & $2(2)$ \\
Mucositis & $12(12)$ & $1(1)$ \\
Neurotoxicity & $3(3)$ & 0 \\
Renal & 0 & 0 \\
Liver & 0 & 0 \\
Fatigue & $11(1)$ & $2(2)$ \\
Cardiac toxicity & & \\
$\quad<50 \%$ LVEF & $5(5)$ & $3(3)$ \\
Congestive failure & 0 & 0 \\
Skin toxicity & $3(3)$ & 0 \\
\hline
\end{tabular}

recorded, occurring in the PET arm. Severe anaemia occurred in one ET patient, as compared to 13 (13\%) PET patients.

Overall, severe gastrointestinal toxicity and fatigue were also more frequent in the PET arm. They caused early chemotherapy discontinuation in a total of eight patients (five PET and three ET). Severe emesis, loss of appetite, and diarrhoea occurred in 10, 13, and $15 \%$ of PET as compared to 4,5 , and $2 \%$ of ET arm. Severe 
fatigue occurred in $11(11 \%)$ patients of PET and two (2\%) patients of ET arm.

Neurotoxicity and mucositis and were also common side effects in both arms. Overall, 34 (34\%) PET and 16 (16\%) ET patients complained of sensory neuropathy, but it was severe in only three patients of PET arm, and in two cases it caused treatment discontinuation. Severe mucositis was recorded in 12 PET patients, and in one case it caused the definitive treatment discontinuation. Only one patient of the ET arm experienced severe mucositis. Renal, liver and cardiac toxicity were very uncommon. There were no cases of severe functional impairment of these organs. Five PET and three ET patients had a LVEF decrease below 50\%. In all but one patient (treated with PET), LVEF returned within the normal range. No patients showed clinical signs of congestive heart failure.

\section{DISCUSSION}

In the present randomised study, we aimed at evaluating whether 12 weekly preoperative cycles of PET with G-CSF support could improve the pCR rate achievable in patients with LABC (stage IIIB) in comparison with ET tri-weekly administration.

Our results seem to suggest that the PET weekly treatment is more effective than the tri-weekly ET regimen in producing a PCR. Indeed, the chance of achieving a complete clearance of the tumour in both breast and axilla in the PET arm was more than twice that of the ET arm.

The rate of pCR obtained in the present study with the standard treatment (ET) does not seem to be underestimated. In the Milan NCI experience, the use of neoadjuvant doxorubicin-paclitaxel combination gave a $7 \%$ pCR rate in a study population including 41 II/IIIA and 38 IIIB patients (Moliterni et al, 1997). Canadian authors recently reported a $9 \%$ pCR rate in 49 patients with locally advanced disease, $60 \%$ of whom had stage IIB and IIIA. They also remarked that, depending on the definition of PCR used, the range of $\mathrm{pCR}$ varied from $4.2 \%$ (using definition from Chevallier) to $10.6 \%$ (using that of NSABP-27 study) (Dent et al, 2005).

The present study was sized setting the pCR rate as the target end point. Of course, the achievement of a complete eradication of the tumour in both breast and axilla may be considered a meaningful end point only if it is associated with a substantial improvement of the long-term disease-free survival.

At the time of the present analysis, the number of observed failures (70 progressions or relapses) is too low to even allow us to speculate about the impact that this new regimen may have on prognosis of these patients. Moreover, given the very small rate of patients who are expected to have a substantial DFS gain (16 vs 6\% pCRs), a much larger study population would be required to statistically detect such a gain.

Analysis of our data strongly suggests that this dose dense approach may be superior only in some subsets of patients and not in the whole population. Indeed, an impressive increase of the pCR rate with the PET regimen was obtained in patients with ER negative (27.5 vs 5.4\%) and/or HER2/neu positive tumour (31 vs $5 \%)$, while the activity of the two regimens was comparable in patients with ER positive (7.5 vs 7\%) and/or HER2/neu negative tumour (10vs 6\%). The modest effect of the primary chemotherapy in ER positive tumours was noted by other investigators, who recently reported a much higher pCR rate with chemotherapy in $\mathrm{ER} / \mathrm{PgR}$ negative patients (Faneyete et al, 2003; Colleoni et al, 2004; Ring et al, 2004; Gianni et al, 2005) and seems to suggest that aggressive chemotherapy should not be advisable in hormonesensitive tumours. In a previous phase II study, testing the PET regimen in patients with large operable breast cancer, we observed a pCR rate in $66 \%$ in ER negative patients as compared to $14 \%$ in ER positive (Frasci et al, 2005).

In the present study, seven out of 22 pCRs relapsed. This relapse rate looks similar to that of the total population. However, in the $\mathrm{ER} / \mathrm{PgR}$ negative group 4/13 pCRs relapsed as compared to $31 / 64$
non-pCRs. Moreover, three out of four relapsing pCRs were also HER2 positive, and in two cases brain was the first site of relapse. These findings suggest that chemotherapy alone is not enough in the management of HER2 positive patients, who might benefit of a prolonged trastuzumab treatment.

On the other hand, the impressive increase of the $\mathrm{PCR}$ rate in $\mathrm{ER} / \mathrm{PgR}$ negative tumours with the weekly treatment could represent a simple 'optical illusion' if not followed by a substantial advantage in long-term disease-free survival of patients. An occult metastatic spread may derive from malignant cell clones, which have developed an acquired resistance to cytotoxic drugs, and are therefore less sensitive to the induction chemotherapy than the primary tumour. At the Mount Sinai Medical Center 144 patients with $L A B C$ treated with neoadjuvant chemotherapy were reviewed to evaluate the prognostic impact of chemotherapy-induced histological changes. Little evidence in favour of predictivity of pathological response was found (Gajdos et al, 2002). In another recent report from the US NCI, 107 patients with stage III breast cancer were treated with a multimodality approach. Pathologic response was not associated with improved survival for stage IIIA and inflammatory breast cancer patients (Low et al, 2004). In an MDACC retrospective analysis conducted on 372 women with LABC, the DFS and OS of the pCRs group were significantly better than those of the other group; however, investigators remarked that a pCR did not eliminate the risk of recurrence (Kuerer et al, 1999).

In the present study, a reduction of the risk of relapse with the PET regimen becomes evident after 3 years in ER/PgR negative patients, and is maintained beyond 5 years. Given the small study population, it is clear that such a difference cannot be statistically significant, even with a more mature follow-up. A new randomised trial, specifically targeted to ER/PgR negative patients, with an adequate sample size is required to address this issue.

The impressive increase of the PCR rate in HER2/neu positive patients with the weekly treatment also deserves some considerations. There are several possible factors responsible for the observed better tumour shrinkage in the PET arm. Firstly, the increase of the epirubicin dose intensity, as previously reported by French authors (Petit et al, 2001).

The adoption of a weekly paclitaxel schedule might also have a role. In the CALGB 9840 trial, (Seidman et al, 2004) weekly paclitaxel significantly improved ORR and survival of metastatic breast cancer patients, when compared with standard every 3 weeks paclitaxel. However, in patients with HER2/neu normal tumour, the weekly administration failed to produce a significant gain in both ORR and survival.

In spite of the very impressive pCR rate observed in HER2 positive patients, the DFS outcome was disappointing in this group, the risk of relapse being relevant even in pCRs. Of course, the discovery of a drug specifically targeting HER2/neu overexpressing tumour cells has deeply modified the therapeutic strategy in this subset of patients. The capability of trastuzumab in improving the prognosis of patients with metastatic disease is well established (Slamon et al, 2001). Preliminary reports also suggest that the addition of trastuzumab to a standard anthracyclinetaxane combination can dramatically increase the pCR rate in women with operable breast cancer (Buzdar et al, 2005).

The addition of trastuzumab to a dose dense aggressive chemotherapy, like PET, could result in a further substantial increment of the pCR rate. Moreover, it could substantially increase the probability of obtaining the eradication of the distant micrometastases, as suggested by the results of several large randomised trials (Piccart-Gebhart et al, 2005; Romond et al, 2005).

A final consideration on the toxicity profiles of the two regimens deserves to be made. Although our dose dense approach did not cause any life-threatening toxicity, it produced a substantial increase of severe side effects, which may negatively impact on 
the quality of life of patients. Severe anaemia was significantly more frequent in the PET arm, and it resulted in a substantially higher proportion of patients complaining about severe fatigue. PET treatment was also associated with more severe nonhaematological toxicity. In particular, diarrhoea, peripheral neuropathy and mucositis occurred more frequently in the PET arm, being the main causes of patient's discomfort. In view of that, an accurate selection of patients who more likely can benefit of this approach is mandatory in the next future.

In conclusion, a 3-month preoperative treatment with weekly PET plus G-CSF support yields a significantly higher pathological

\section{REFERENCES}

Bear HD, Anderson S, Brown A, Smith R, Mamounas EP, Fisher B, Margolese R, Theoret H, Soran A, Wickerham DL, Wolmark N, National Surgical Adjuvant Breast and Bowel Project protocol B-27 (2003) The effect on tumor response of adding sequential preoperative docetaxel to preoperative doxorubicin and cyclophosphamide: preliminary results from the National Surgical Adjuvant Breast and Bowel Project protocol B-2. J Clin Oncol 21: 4165-4174

Buzdar AU, Ibrahim NK, Francis D, Booser DJ, Thomas ES, Theriault RL, Pusztai L, Green MC, Arun BK, Giordano SH, Cristofanilli M, Frye DK, Smith TL, Hunt KK, Singletary SE, Sahin AA, Ewer MS, Buchholz TA, Berry D, Hortobagyi GN (2005) Significantly higher pathologic complete remission rate after neoadjuvant therapy with trastuzumab, paclitaxel, and epirubicin chemotherapy: results of a randomized trial in human epidermal growth factor receptor 2-positive operable breast cancer. J Clin Oncol 23: 3676-3685

Casagrande JT, Pike MC, Smith PG (1978) An improved formula for calculating sample sizes for comparing two binomial distributions. Biometrics 34: $483-486$

Chevallier B, Roche H, Olivier JP, Chollet P, Hurteloup P (1993) Inflammatory breast cancer. Pilot study of intensive induction chemotherapy (FEC-HD) results in a high histologic response rate. Am J Clin Oncol 16: 223-228

Chollet P, Amat S, Cure H, de Latour M, Le Bouedec G, Mouret-Reynier MA, Ferriere JP, Achard JL, Dauplat J, Penault-Llorca F (2002) Prognostic significance of a complete pathological response after induction chemotherapy in operable breast cancer. $\mathrm{Br} J$ Cancer 86: $1041-1046$

Colleoni M, Viale G, Zahrieh D, Pruneri G, Gentilini O, Veronesi P, Gelber RD, Curigliano G, Torrisi R, Luini A, Intra M, Galimberti V, Renne G, Nole F, Peruzzotti G, Goldhirsch A (2004) Chemotherapy is more effective in patients with breast cancer non expressing steroid hormone receptors: a study of preoperative treatment. Clin Cancer Res 10: $6622-6628$

Dent RA, Verma S, Fitzgerald B, Halloway C, Lickley L, Kahn H, Wright F, Pignol JP, Trudeau M, Lenis M, Clemons M (2005) Do clinical trials of neoadjuvant chemotherapy for locally-advanced breast cancer overestimate the actual response rates observed in clinical practice? J Clin Oncol 23: 100s (abstr. 890)

Dieras V, Fumoleau P, Romieu G, Tubiana-Hulin M, Namer M, Mauriac L, Guastalla JP, Pujade-Lauraine E, Kerbrat P, Maillart P, Penault-Llorca F, Buyse M, Pouillart P (2004) Randomized parallel study of doxorubicin plus paclitaxel and doxorubicin plus cyclophosphamide as neoadjuvant treatment of patients with breast cancer. J Clin Oncol 22: $4958-4965$

Dombernowsky P, Gehl J, Boesgaard M, Paaske T, Jensen BV (1996) Doxorubicin and paclitaxel, a highly active combination in the treatment of metastatic breast cancer. Semin Oncol 23: 23-27

Ezzat AA, Ibrahim EM, Ajarim DS, Rahal MM, Raja MA, Tulbah AM, Al-Malik OA, Al-Shabanah M, Sorbris R (2004) Phase II study of neoadjuvant paclitaxel and cisplatin for operable and locally advanced breast cancer: analysis of 126 patients. Br J Cancer 90: 968-974

Faneyete IF, Schrama JG, Peterse JL, Remijnse PL, Rodenhuis S, van Vijver MJ (2003) Breast cancer response to neoadjuvant chemotherapy: predicting markers and relation with outcome. Br J Cancer 88: 406-412

Fisher B, Brown A, Mamounas E, Wieand S, Robidoux A, Margolese RG, Cruz Jr AB, Fisher ER, Wickerham DL, Wolmark N, DeCillis A, Hoehn JL, Lees AW, Dimitov NV (1997) Effect of preoperative chemotherapy on loco-regional disease in women with operable breast cancer: Findings complete response rate in women with LABC compared with four tri-weekly ET cycles. The superiority of the PET regimen was limited to hormone-receptor negative or HER2/neu overexpressing tumours. PET treatment was associated with a substantial increase in nonhaematological toxicity. A longer follow-up is needed to better evaluate the impact of this new approach on failure-free and OS. However, such a dose-dense approach is not recommended in women with ER/PgR positive LABC. Adequately powered, randomised trials are required to evaluate whether this approach can significantly improve prognosis of ER negative and/or HER2 positive LABC patients.

from National Surgical Adjuvant Breast and Bowel Project B-18. J Clin Oncol 15: $2483-2493$

Frasci G, D'Aiuto G, Comella P, Apicella A, Thomas R, Capasso I, Di Bonito M, Carteni G, Biglietto M, De Lucia L, Maiorino L, Piccolo S, Bianchi U, D'Aniello R, Lapenta L, Comella G (1999) Cisplatin-epirubicin-paclitaxel weekly administration in advanced breast cancer: A phase I study of the Southern Italy Cooperative Oncology Group. Breast Cancer Res and Treat 56: 239-252

Frasci G, D’Aiuto G, Comella P, Thomas R, Botti G, Di Bonito M, D’Aiuto M, Romano G, Rubulotta MR, Comella G (2005) A two-month cisplatinepirubicin-paclitaxel weekly administration is highly effective in large operable breast cancer. A SICOG phase II study Ann Oncol 16: $1268-1275$

Frasci G, D'Aiuto G, Comella P, Thomas R, Capasso I, Di Bonito M, Rivellini F, Carteni G, De Lucia L, Maiorino L, D’Aniello R, Frezza P, Lapenta L, Comella G (2000) Cisplatin-epirubicin-paclitaxel weekly administration with G-CSF support in advanced breast cancer: A Southern Italy Cooperative Oncology Group. Phase II study. Breast Cancer Res Treat 62: 87-97

Gajdos C, Tartter PI, Estabrook A, Gistrak MA, Jaffer S, Bleiweiss I (2002) Relationship of clinical and pathologic response to neoadjuvant chemotherapy and outcome of locally advanced breast cancer. J Surg Oncol 80: 4-11

Gianni L, Baselga J, Eiermann W, Guillem Porta V, Semiglazov V, Lluch A, Zambetti M, Sabadell D, Raab G, Llombart Cussac A, Bozhok A, MartinezAgullo A, Greco M, Byakhov M, Lopez Lopez JJ, Mansutti M, Valagussa P, Bonadonna G, European Cooperative Trial in Operable Breast Cancer Study Group (2005) Feasibility and tolerability of sequential doxorubicin/ paclitaxel followed by cyclophosphamide, methotrexate, and fluorouracil and its effects on tumor response as preoperative therapy. Clin Cancer Res 11: $8715-8721$

Green MC, Buzdar A, Smith T, Ibrakim NK, Valero V, Rosales MF, Cristofanili M, Booser DJ, Pusztai L, Rivera E, Theriault RL, Carter C, Frye D, Hunt KK, Symmans WF, Strom EA, Sahin AA, Sikov W, Hortobagyi GN (2005) Weekly paclitaxel improves pathologic complete remission in operable breast cancer when compared with paclitaxel once every 3 weeks. J Clin Oncol 23: $5983-5992$

Hortobagyi G, Buzdar AU (1997) Locally-advanced breast cancer. In: Textbook of breast cancer: A clinical guide to therapy Bonadonna G, Hortobagyi GN, Gianni AM (eds) pp 155-168. London, UK: Martin Dunitz Ltd

Kaplan ES, Meier P (1958) Non parametric estimation for incomplete observations. J Am Stat Assoc 53: $557-580$

Kuerer HM, Newman LA, Smith TL, Ames FC, Hunt KK, Dhingra K, Theriault RL, Singh G, Binkley SM, Sneige N, Buchholz TA, Ross MI, McNeese MD, Buzdar AU, Hortobagyi GN, Singletary SE (1999) Clinical course of breast cancer patients with complete pathological primary tumor and axillary lymph node response to doxorubicin-based neoadjuvant chemotherapy. J Clin Oncol 17: 460-469

Low JA, Berman AW, Steinberg SM, Danforth DN, Lippman ME, Swain SM (2004) Long-term follow-up for locally-advanced and inflammatory breast cancer patients treated with multimodality therapy. J Clin Oncol 22: $4067-4074$

Miller AB, Hoogstraten B, Staquet M, Winkler A (1981) Reporting results of cancer treatment. Cancer 47: $207-214$

Moliterni A, Tarenzi E, Capri G, Terenziani M, Bertuzzi A, Grasselli G, Agresti R, Piotti P, Greco M, Salvadori B, Pilotti S, Lombardi F, Valagussa P, Bonadonna G, Gianni L (1997) Pilot study of primary chemotherapy 
with doxorubicin plus paclitaxel in women with locally advanced or operable breast cancer. Semin Oncol 24, S17-10-S17-14

Orlando L, Colleoni M, Curigliano G, Nole F, Ferretti G, Masci G, Peruzzotti G, Minchella I, Intra M, Veronesi P, Viale G, Goldhirsch A (2001) Chemotherapy with vinorelbine, cisplatin and continuous infusion of 5-fluorouracil in locally advanced breast cancer: a promising low-toxic regimen. Anticancer Res 21: 4135-4139

Petit T, Borel C, Ghnassia JP, Rodier JF, Escande A, Mors R, Haegele P (2001) Chemotherapy response of breast cancer depends on HER2 and anthracycline dose intensity in the neoadjuvant setting. Clin Cancer Res 7: $1577-1581$

Piccart-Gebhart MJ, Procter M, Leyland-Jones B, Goldhirsch A, Untch M, Smith I, Gianni L, Baselga J, Bell R, Jackisch C, Cameron D, Dowsett M, Barrios CH, Steger G, Huang CS, Andersson M, Inbar M, Lichinitser M, Lang I, Nitz U, Iwata H, Thomssen C, Lohrisch C, Suter TM, Ruschoff J, Suto T, Greatorex V, Ward C, Straehle C, McFadden E, Dolci MS, Gelber RD, Herceptin Adjuvant (HERA) Trial Study Team (2005) Trastuzumab after adjuvant chemotherapy in HER2-positive breast cancer. $N$ Engl J Med 353(16): 1659-1672

Ring AE, Smith IE, Ashley S, Fulford LG, Lakhani SR (2004) Oestrogen receptor status, pathological complete response and prognosis in patients receiving neoadjuvant chemotherapy for early breast cancer. Br J Cancer 91: 2012-2017
Romond EH, Perez EA, Bryant J, Suman VJ, Geyer Jr CE, Davidson NE, Tan-Chiu E, Martino S, Paik S, Kaufman PA, Swain SM, Pisansky TM, Fehrenbacher L, Kutteh LA, Vogel VG, Visscher DW, Yothers G, Jenkins RB, Brown AM, Dakhil SR, Mamounas EP, Lingle WL, Klein PM, Ingle JN, Wolmark N (2005) Trastuzumab plus adjuvant chemotherapy for operable HER2-positive breast cancer. $N$ Engl J Med 353(16): $1673-1684$

Seidman AD, Berry D, Cirrincione C, Harris L, Dressler L, Muss H, Norton L, Winer E, Hudis C (2004) CALGB 9840: Phase III study of weekly paclitaxel via 1-hr infusion versus standard infusion every third week in the treatment of metastatic breast cancer, with trastuzumab for HER2 positive MBC and randomized for Taxol in HER2 normal MBC. J Clin Oncol 22: 6s (abstr. 512)

Slamon DJ, Leyland-Jones B, Shak S, Fuchs H, Paton V, Bajamonde A, Fleming T, Eiermann W, Wolter J, Pegram M, Baselga J, Norton L (2001) Use of chemotherapy plus a monoclonal antibody against HER2 for metastatic breast cancer that overexpresses HER2. New Engl J Med 344: $783-792$

Smith IC, Heys SD, Hutcheon AW, Miller ID, Payne S, Gilbert FJ, Ah-See AK, Eremin O, Walker LJ, Sarkar JK, Eggleton SP, Ogston KN (2002) Neoadjuvant chemotherapy in breast cancer: Significantly enhanced response with docetaxel. J Clin Oncol 20: $1456-1466$ 